\title{
Streptococcus pneumoniae aislados durante 2002-2006: serotipos y resistencia antibiótica. Correlación con las vacunas existentes
}

\author{
Streptococcus pneumoniae isolated during 2002-2006: serotypes and antibiotics resistance. \\ Correlation with existing vaccines
}

\author{
José María Guevara-Duncan ${ }^{1}$, Asunción Fenoll ${ }^{2}$, Esther Valencia ${ }^{1}$, Rito Zerpa 1,3, \\ José María M. Guevara-Granados 1,4 \\ ${ }^{1}$ Instituto de Medicina Tropical Daniel A. Carrión, Facultad de Medicina, UNMSM. \\ ${ }^{2}$ Centro Nacional de Microbiología, Instituto de Salud Carlos III, Majadahonda, Madrid, España. \\ ${ }^{3}$ Instituto de Salud del Niño. \\ ${ }^{4}$ Hospital Nacional Daniel A. Carrión, Callao.
}

\begin{abstract}
Resumen
Objetivos: Identificar los serotipos de S. pneumoniae aislados, correlacionándolos con los incluidos en las vacunas existentes y su resistencia antimicrobiana. Diseño: Estudio descriptivo, observacional y longitudinal. Lugar: Instituto de Medicina Tropical Daniel A. Carrión, Facultad de Medicina, UNMSM. Material biológico: Cepas de Streptococcus pneumoniae. Intervenciones: Cuarenta Streptococcus pneumoniae de nuestro cepario, aislados entre el 2002 y 2006 , fueron serotipificados en el Instituto de Salud Carlos III en Madrid -España; 15 fueron invasivos, 11 aislados de infecciones localizadas, 6 de portadores y 8 eran multiresistentes. Principales medidas de resultados: Protección de las vacunas existentes en nuestro medio a las infecciones causadas por Streptococcus pneumoniae. Resultados: Hubo 14 serotipos diferentes y los serogrupos más identificados fueron 23,19 y 6 . El 28,6\% estaba contenido en la vacuna 7-valente, $42,9 \%$ en la 9-valente, $50 \%$ en la 11 -valente y el $71,4 \%$ en la 23 -valente; $57,5 \%$ fue resistente a la penicilina y $30 \%$ a eritromicina. El grupo de Streptococcus invasivo resultó más sensible a los antibióticos que los otros grupos. Los serotipos asociados a multirresistencia fueron $19 \mathrm{~F}$ y $23 \mathrm{~F}$. Conclusiones: Ninguna de las vacunas protege a todas las infecciones causadas por Streptococcus pneumoniae, en nuestro medio.
\end{abstract}

Palabras clave: Streptococcus pneumoniae; pruebas serológicas; farmacorresistencia bacteriana.

\begin{abstract}
Objectives: To identify isolated S. pneumoniae serotypes correlating them with those covered by existing vaccines and determining antimicrobial resistance. Design: Descriptive, observational and longitudinal study. Setting: Daniel A Carrion Tropical Medicine Institute, School of Medicine, UNMSM. Biologic material: Streptococcus pneumoniae stocks. Interventions: Forty Streptococcus pneumoniae stocks isolated between 2002 and 2006 were serotyped at Carlos III Health Institute in Madrid, Spain; 15 were invasive, 11 isolated from localized infections, 6 from carriers and 8 were multiresistant. Main outcome measures: Protection of local existing vaccines to Streptococcus pneumoniae infections. Results: There were 14 different serotypes and most identified groups were 23, 19 and 6, 28,6\% contained in the 7 -valent vaccine, $42,9 \%$ in the 9 -valent, $50 \%$ in the 11 -valent and $71,4 \%$ in the 23 -valent; $57,5 \%$ were resistant to penicillin and $30 \%$ to erythromycin. The invasive Streptococcus group proved to be more sensitive to antibiotics than the other groups; $19 \mathrm{~F}$ and $23 \mathrm{~F}$ serotypes were multiresistant. Conclusions: In our environment none of the studied vaccines protected all the infections caused by Streptococcus pneumoniae. Resistance to penicillin was high.
\end{abstract}

Key words: Streptococcus pneumoniae; serologic tests; drug resistance, bacterial.

\section{INTRODUCCIÓN}

Streptococcus pneumoniae es el patógeno aislado con mayor frecuencia en infecciones focales, como otitis media aguda y sinusitis, y el más frecuentemente relacionado con las infecciones invasivas en la infancia, las cuales constituyen un serio problema de salud pública en países en desarrollo, por las elevadas tasas de mortalidad que presentan (1). La nasofaringe es el principal reservorio de $S$. pneumoniae y el estado de portador precede en la mayoría de los casos a una infección neumocócica ${ }^{(2)}$.

La vacuna polisacárida antineumocócica 23-valente, en uso desde 1982, no genera respuesta inmunológica en niños menores de 2 años. Se ha desarrollado vacunas conjugadas antineumocócicas 7-valente, 9-valente, 11 valente, las cuales son inmunogénicas para los serotipos contenidos y pueden ser usadas en menores de 2 años, los cuales disminuyen la colonización nasofaríngea ${ }^{(3)}$. Al respecto, se ha descrito 90 serotipos de S. pneumoniae y sería prácticamente imposible incluir a todos en una vacuna conjugada ${ }^{(1)}$.

Un problema que complicó el manejo de las infecciones neumocócicas es el incremento de la resistencia a penicilina y otros antibióticos, habiendo diferencia regionales en la diseminación de la resistencia.

Cada región geográfica de los países se preocupa por controlar los serotipos de neumococos aislados en el tiempo, porque pueden variar y es necesario compararlos con las vacunas existentes, para conocer el grado de protección que tendrán en cada región o país. Así mismo, el tratamiento de elección para las infecciones neumocócicas fue la penicilina, pero el aumento de su resistencia obliga a vigilar la resistencia a otros antibióticos que podrían reemplazarla en caso de emergencia.

El objetivo del presente trabajo fue identificar los serotipos de 40 cepas de $S$. pneumoniae de nuestros aislamientos, así como correlacionarlos con las vacunas existentes en la actualidad, a fin de determinar si tienen la cobertura necesaria para los serotipos encontrados. Asimismo, determinar la resistencia antibiótica de los serotipos identificados. 


\section{MATERIALES Y MÉTODOS}

Se realizó un estudio de tipo descriptivo, observacional y longitudinal, utilizándose para ello 40 cepas de Streptococcus pneumoniae de nuestro cepario del Instituto de Medicina Tropical de la Facultad de Medicina de San Fernando, UNMSM, aislados entre los años 2002 y 2006, las cuales fueron serotipificados en el Instituto de Salud Carlos III, en Madrid, España.

De 332 cepas aisladas, criopreservadas y viables a la resiembra, seleccionamos 50 de forma aleatoria, por ser el número máximo ofrecido para tipificar: 15 invasivas, 15 causantes de infección localizada, 10 aisladas de portadores y 10 multirresistentes (resistentes a 4 o más antibióticos), las cuales fueron reconfirmadas con las pruebas de optochin y la bilis-solubilidad, para luego nuevamente ser congeladas en crioviales con leche descremada (skim milk).

Para el envío de las cepas a España, se descongeló las 50 cepas y se las sembró en placas con agar Columbia, sangre de carnero, e incubadas por 24 horas a $37^{\circ} \mathrm{C}$. Todo el desarrollo de cada una se cosechó con hisopo, que inmediatamente se introdujo en tubos plásticos con el medio de transporte Stuart marca COPAN, de manufactura italiana. El mismo día, la empresa de transporte autorizada recogió los tubos, los acondicionó conforme a las normas internacionales establecidas para transporte de cepas, llevándoselos para mantenerlos en medio ambiente hasta su llegada al Instituto de Salud Carlos III en Madrid, España.

La confirmación de las cepas, en España, se hizo con la susceptibilidad al optochin y la solubilidad al desoxicolato de sodio. El serotipado se realizó por la reacción de Quellung, con antisueros proporcionados por Statens SERUM Institute (Copenhague, Dinamarca). El antibiograma de las cepas se hizo por el método de disco difusión estandarizado, de acuerdo a las normas del CLSI (antes NCCLS) (4).

De las 50 cepas, se eliminó 10: dos por no desarrollar al llegar a España, 4 por no ser tipificables y 4 por no ser confirmadas como neumococos. Así, las cepas se redujeron a 40: 15 invasivas, 11 de infecciones localizadas, 8 multiresistentes y 6 de portadores

Los datos recolectados fueron consignados en una base de datos y para la muestra de resultados se utilizó la estadística descriptiva.

\section{RESULTADOS}

La identificación completa de los serotipos encontrados se aprecia en la Tabla 1. Es así
Tabla 1. Características y serotipos de las cepas estudiadas

\begin{tabular}{|c|c|c|c|c|c|}
\hline Código & Año & Edad & Sexo & Muestra & Serotipo \\
\hline \multicolumn{6}{|c|}{ Cepas multirresistentes } \\
\hline MR 1 & 2003 & 3 años & M & Hisopado nasal & No neumococo \\
\hline MR 2 & 2004 & 1 año & M & Sangre & $19 \mathrm{~F}$ \\
\hline MR 3 & 2004 & 9 meses & $M$ & Secreción nasal & $19 F$ \\
\hline MR 4 & 2004 & 3 años & M & Hisopado nasal & $23 \mathrm{~F}$ \\
\hline MR 5 & 2004 & 7 años & M & Secreción nasal & $23 \mathrm{~F}$ \\
\hline MR 6 & 2003 & 2 años & $\mathrm{F}$ & Secreción nasal & $23 \mathrm{~F}$ \\
\hline MR 7 & 2004 & 1 mes & $\mathrm{F}$ & Secreción ocular & $23 \mathrm{~F}$ \\
\hline MR 8 & 2004 & 5 años & M & Hisopado nasal & Crec negativo \\
\hline MR 9 & 2004 & 5 años & $\mathrm{F}$ & Hisopado nasal & $19 F$ \\
\hline MR 10 & 2004 & 4 años & M & Secreción nasal & $19 F$ \\
\hline
\end{tabular}

\section{Cepas Invasivas}

I-1 $2003 \quad 16$ años $\quad F \quad$ Líquido cefalorraquideo

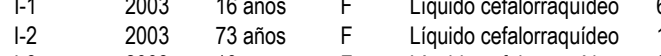

I-4 $2003 \quad 5$ meses M Líquido cefalorraquídeo

I-5 $2003 \quad 50$ años M Líquido pleural $\quad 6 \mathrm{~A}$

I-6 $2003 \quad 71$ años $F \quad$ Líquido pleural

I-7 $2004 \quad 45$ años $\quad M \quad$ Líquido cefalorraquídeo 3

I-8 $2004 \quad 1$ mes $\quad M \quad$ Líquido cefalorraquideo 5

I-9 $2003 \quad 2$ años $M \quad$ Sangre

I-10 $2003 \quad 2$ años $F \quad$ Sangre

I-11 $2003 \quad 1$ año $\quad M \quad$ Sangre

$\begin{array}{lllll}\mathrm{I}-12 & 2003 & 7 \text { meses } & \mathrm{F} & \text { Sangre }\end{array}$

I-13 $2004 \quad 4$ meses M Sangre 34

I-14 $2004 \quad 4$ años $\quad F \quad$ Sangre 38

I-15 20047 años M Sangre 6B

Cepas de infecciones localizadas

IL-1 $2004 \quad 18$ años $\quad F \quad$ Secreción ótica

IL-2 $2004 \quad 4$ años $\quad M \quad$ Secreción nasal

IL-3 $2004 \quad 26$ años $F \quad$ Esputo

IL-4 $2004 \quad 6$ años $\quad M \quad$ Secreción faríngea

IL-5 $2004 \quad 2$ años $F \quad$ Secreción ótica

IL-6 $2003 \quad 6$ años $\quad F \quad$ Secreción nasal

IL-7 $2003 \quad 10$ meses $M \quad$ Secreción nasal

IL-8 $2003 \quad 3$ años $\quad M \quad$ Secreción nasal

IL-9 $2004 \quad 3$ meses $M \quad$ Secreción nasal

IL-10 $2004 \quad 12$ años M Secreción faríngea

IL-11 $2004 \quad 9$ meses M Secreción nasal

IL-12 $2006 \quad 3$ años $\quad M \quad$ Secreción nasal

IL-13 $2004 \quad 8$ años $M \quad$ Secreción nasal

IL-14 $2003 \quad 8$ años $\quad F \quad$ Secreción nasal

IL-15 $2003 \quad 12$ años

Cepas de portadores

P-1 $2004 \quad 1$ año

P-2 $2004 \quad 1$ año

P-3 2004 1año

P-4 $2004 \quad 2$ años

P-5 $2004 \quad 2$ años

P-6 20042 años

P-7 $2004 \quad 3$ años

P-8 $2004 \quad 3$ años

P-9 $2004 \quad 3$ años

P-10 $2004 \quad 4$ años

$M$
$M$
$M$
$F$
$F$
$F$
$M$
$M$
$F$

Secreción nasal

Hisopado nasal

Hisopado nasal

Hisopado nasal

Hisopado nasal

Hisopado nasal

Hisopado nasal

Hisopado nasal

Hisopado nasal

Hisopado nasal

Hisopado nasal
I-3 $2003 \quad 10$ meses $F \quad$ Líquido cefalorraquídeo $6 A$

3, 6A, 6B, 8, 13, 14, 19F, 23F; de ellos, 38 (44,4\%) se encontraban contenidos en la vacuna 7 -valente, $44,4 \%$ en la 9- valente, $55 \%$ en la 11 -valente y $66,6 \%$ en la 23-valente.

Los Streptococcus pneumoniae invasivos serotipados fueron 16 , incluyendo uno multirresistente; los serotipos identificados fueron: 1,3, 5, 6A, 6B, 8, 14, 19F, 23F, 34 y 38 , es decir, 11 serotipos diferentes, de los cuales dentro de la vacuna 7-valente se encontró el 36,3\%, en la 9-valente $54,5 \%$, en la $11-$ valente $63,3 \%$ y dentro de la 23-valente $72,7 \%$.

Si separamos las cepas invasivas por edad del paciente, los serotipos identificados en niños de 2 años o menos fueron: 1 , $5,6 \mathrm{~A}, 14,19 \mathrm{~F}, 23 \mathrm{~F}$ y 34 , $42,8 \%$ de los cuales estuvieron dentro de la vacuna 7 -valente, $71,4 \%$ en la 9 -valente y $71,4 \%$ dentro de la 11-valente. Los serotipos invasivos de los mayores de 2 años fueron: $3,6 \mathrm{~A}, 6 \mathrm{~B}, 8,14,38,16 \%$ de los cuales dentro de la vacuna 7 -valente, $33,3 \%$ en la 9 -valente, $33,3 \%$ en la $11-$ valente y $66,6 \%$ en la 23-valente. Dentro de los 8 neumococos multirresistentes se identificó solo 2 serotipos: $19 \mathrm{~F}$ y $23 \mathrm{~F}$.

En la Tabla 2 se puede apreciar la resistencia a que los serotipos identificados más frecuentemente fueron los serotipos $23(27,5 \%), 19$ (20\%), $6(20 \%)$ y $14(7,5 \%)$. Dentro de las cepas estudiadas se identificó 14 serotipos diferentes, de los cuales $28,6 \%$ se encontraban en la vacuna 7 -valente; $42,9 \%$ dentro de la 9-valente, $50 \%$ en la 11 -valente y $71,4 \%$ en la vacuna 23 -valente.

En los niños menores de 2 años de edad, se identificó los siguientes serotipos: 1,5 , $6 \mathrm{~A}, 15 \mathrm{~B}, 19 \mathrm{~A}, 19 \mathrm{~F}, 23 \mathrm{~F}$ y 34 ; de los cuales $25 \%$ se encontraban contenidos en la vacuna 7 -valente, $50 \%$ en la 9 -valente y $50 \%$ en la 11-valente. En los niños mayores de 2 años y adultos, se identificó los siguientes serotipos: los antibióticos de los 40 Streptococcus pneumoniae. La resistencia más alta fue para trimetoprim-sulfametoxazol, con $77,5 \%$, seguido de la penicilina, con $57,5 \%$; solo vancomicina no tuvo resistencia. Las cepas invasivas fueron menos resistentes, independientemente del serotipo (Tabla 3). Los principales serotipos asociados a resistencia a la penicilina -incluyendo intermedios- ( $\mathrm{Ta}$ bla 4) fueron 23F y 19F. Los serotipos principales asociados a resistencia a los macrólidos -incluyendo intermedios- (Tabla 5) fueron $19 \mathrm{~F}$ y $23 \mathrm{~F}$. Asimismo, en la Tabla 6 podemos apreciar la resistencia por serotipos de los 40 Streptococcus pneumoniae estudiados. 
Tabla 2. Resistencia a los antimicrobianos de los 40 Streptococcus pneumoniae en orden decreciente (incluyendo intermedios)

\begin{tabular}{lc}
\hline Antimicrobiano & $\begin{array}{c}\text { Porcentaje de } \\
\text { resistencia }\end{array}$ \\
\hline Trimetoprim - Sulfametoxazol & 77,5 \\
Penicilina & 57,5 \\
Tetraciclina & 50,0 \\
Doxiciclina & 30,0 \\
Eritromicina & 30,0 \\
Cloranfenicol & 30,0 \\
Ciprofloxacino & 17,5 \\
Lincomicina & 12,5 \\
Cefalotina & 2,5 \\
Cefotaxima & 2,5 \\
Ceftriaxona & 2,5 \\
Cefuroxima & 2,5 \\
Rifampicina & 2,5 \\
Vancomicina & 0 \\
\hline
\end{tabular}

\section{DISCUSIÓN}

Aunque en el presente trabajo son pocas las cepas de Streptococcus pneumoniae serotipadas, podemos correlacionarlas con otros estudios. Los serogrupos más numerosos, en nuestras cepas, de todos los orígenes fueron el 23, 19 y 6, coincidiendo con lo informado por Fenoll, en España ${ }^{(5)}$. Comparando los serogrupos identificados con los contenidos en la vacuna 7 -valente, solo $28,6 \%$ están contenidos, menos que los encontrados por Cullota y col., en portadores ${ }^{(6)}$.

Los serogrupos pediátricos hallados en las comunicaciones son el 6, 9, 14, 19 y 23 (7) y nosotros hemos encontrado, además, el 1, 5, 15 y 34. En España (3), los serogrupos frecuentemente aislados de niños fueron 6 , 14,19 y 23 , coincidiendo en los serogrupos 6,19 y 23 , pero teniendo además el $1,5,15$ y 34 . También, se ha considerado serogrupos invasivos en estudios previos ${ }^{(8)}-1,3$, $4,5,6,7,9,14,18,19,23$ y 24 -y dentro de ellos nosotros hemos identificado el 1, 3, 5 , 6,14 y 23, y además los serogrupos 8,34 y 38 , estando el $36,3 \%$ dentro de la vacuna 7-valente.
Tabla 3. Resistencia de los 15 Streptococcus pneumoniae invasivos.

\begin{tabular}{lc}
\hline Antimicrobiano & $\begin{array}{c}\text { Porcentaje de } \\
\text { resistencia }\end{array}$ \\
\hline Trimetoprim - Sulfametoxazol & 53,30 \\
Penicilina & 33,33 \\
Cloranfenicol & 6,66 \\
Eritromicina & 6,66 \\
Tetraciclina & 6,66 \\
Cefalotina & 0 \\
Cefotaxima & 0 \\
Ceftriaxona & 0 \\
Cefuroxima & 0 \\
Ciprofloxacina & 0 \\
Lincomicina & 0 \\
Rifampicina & 0 \\
Doxiciclina & 0 \\
Vancomicina & 0 \\
\hline
\end{tabular}

Considerando los serotipos más frecuentes de portadores o colonizados, se ha informado sobre 6 serotipos ${ }^{(2,8)}$ y 5 serotipos ${ }^{(9)}$; dos serotipos (6A y 15B) de portadores nuestros no estaban en esos grupos.

La resistencia a la penicilina de los 40 Streptococcus pneumoniae estudiados fue de $57,5 \%$. Otros estudios realizados en el Perú han informado como sigue: Grupo Multifuncional de Neumonías ${ }^{(10)} 23,1 \%$, en 2003; Guevara y col ${ }^{(11)}, 62 \%$ en aislamientos de 2002 y $44 \%$ en aislamientos de 2003; Cullota y col ${ }^{(6)}$, en 2002, susceptibilidad reducida a la penicilina $-15,1 \%$ en aislamientos de nasofaringe y $42,9 \%$ en aislamientos clínicos-; Guevara y col ${ }^{(12)}$, en 2004, 31,3\%; y, Ochoa y col ${ }^{(13)}$ comunicaron una resistencia de $58 \%$, en el 2007.

La resistencia a la penicilina halladas en otros países: Fenoll y col (5), en España, 49\%; Syrogiannopoulos y col ${ }^{(6)}$, en Grecia, $16 \%$; Schettinii y col ${ }^{(9)}$, en Italia, $8,62 \%$; Sener y col ${ }^{(14)}$, en Turquía, 54,17\%.

Lamentablemente, no tuvimos acceso a los E-tests recomendados para confirmar resultados del método disco-difusión. Los

\begin{tabular}{cc}
$\begin{array}{c}\text { Tabla 4. Serotipos asociados a resistencia a la } \\
\text { penicilina (incluyendo intermedios). }\end{array}$ \\
\hline Serotipo & $\begin{array}{c}\text { Resitentes a } \\
\text { penicilina }\end{array}$ \\
\hline $23 \mathrm{~F}$ & 9 \\
$19 \mathrm{~F}$ & 6 \\
14 & 3 \\
$6 \mathrm{~B}$ & 1 \\
13 & 1 \\
3 & 1 \\
$6 \mathrm{~A}$ & 1 \\
34 & 1 \\
\hline & \\
Tabla 5. Serotipos asociados a resistencia a los \\
macrólidos (incluyendo intermedios). \\
\hline Serotipo & Resitentes a \\
\multicolumn{1}{c}{ macrólidos } \\
\hline $19 \mathrm{~F}$ & 5 \\
$23 \mathrm{~F}$ & 3 \\
$6 \mathrm{~A}$ & 2 \\
$6 \mathrm{~B}$ & 1 \\
13 & 1 \\
\hline
\end{tabular}

serotipos que encontramos asociados a la resistencia a penicilina fueron principalmente 23F y 19F, también encontrados en España (5). La resistencia a los macrólidos (eritromicina) de los 40 Streptococcus pneumoniae estudiados fue de $30 \%$, un poco más alto que lo encontrado en España ${ }^{(5)}$ y menor a lo hallado en Italia ${ }^{(9)}$; los principales serotipos asociados a la resistencia a los macrólidos fueron $19 \mathrm{~F}$ y $23 \mathrm{~F}$, coincidiendo con Italia (9), en el serogrupo 19.

Los serotipos $19 \mathrm{~F}$ y $23 \mathrm{~F}$ acapararon nuestras cepas multirresistentes; es reconocido que el serotipo $19 \mathrm{~F}$ es multidrogo-resistente (5). Llama la atención que el serotipo $23 \mathrm{~F}$ lo aislamos de sangre (invasivo) en un caso, resultando sensible a todos los antibióticos enfrentados.

En conclusión, según lo hallado en el presente trabajo, variados serotipos circulan por nuestros pacientes. La vacuna 7-Valente, aunque con un bajo contenido de los serotipos que tenemos, es recomendable emplearla en niños menores de 2 años, porque en algo disminuirá la incidencia, hasta que ingresen a nuestro país las vacunas 9-valente y 11-valente. En los niños mayores de 2 años, se debería usar la vacuna 23-Valente, porque cubre más a los serotipos de Streptococcus pneumoniae identificados en nuestras cepas. La resistencia a la penicilina sigue aumentando.

\section{AGRADECIMIENTO}

A la Facultad de Medicina de San Fernando por su apoyo económi- 
co, a través del 4to. Concurso de Proyectos de Investigación.

\section{REFERENCIAS BIBLIOGRÁFICAS}

1. Ruvinsky R, Gentile A, Regueira M, Corso A, Pace J, Bakir J y col. Infecciones invasivas por Streptococcus pneumoniae: Estudio epidemiológico e importancia del desarrollo de un sistema de vigilancia. Rev Chil Pediatr. 2004;75(1):77-9.

2. Syrogiannopoulos GA, Katopodis GD, Grivea JN, Beratis NG. Antimicrobial use and serotype distribution of nasopharyngeal Streptococcus pneumoniae isolates recovered from Greek children younger than 2 years old. Clin Infect Dis. 2002; 35(10):1174-82.

3. Finkelstein JA, Huang SS, Daniel J, Rifas-Shiman SL, Kleinman K, Goldmann D, et al. Antibiotic-resistant Streptococcus pneumoniae in the heptavalent pneumococcal conjgate vaccine era: Predictors of carriage in a multicommunity simple. Pediatrics. 2003;112(4):862.

4. Kiehlbauch JA, Hannett GE, Salfinger M, Archinal W, Monserrat C, Carlyn C. Use of the National Committee for Clinical Laboratory Standards Guidelines for Disk Diffusion Susceptibility Testing in New York State Laboratories. J Clin Microbiol. 2000;38(9):3341-8.

5. Fenoll A, Jado I, Vicioso D, Pérez A, Casal J. Evolution of Streptococcus pneumoniae serotypes and antibiotic resistance in Spain: Update (1990 to 1996). J Clin Microbiol. 1998;36(12):3447-54.
6. Cullotta AR, Kalter HD, Delgado J, Gilman RH, Facklam RR, et al. Antimicrobial.

susceptibilities and serotype distribution of Streptococcus pneumoniae isolates from a low socioeconomic area in Lima, Peru. Clin Diag Lab Inmunol. 2002;9(6):1328-31.

7. Madhi SA, Peterson K, Madhi A, Wasas A, Klugman KP. Impact of human immunodeficiency virus type 1 on the disease spectrum of Streptococcus pneumoniae in South African children. Pediatric Infectious Disease J. 2000;19(12):1141-7.

8. Marchisio P, Esposito S, Schito GC, Marchese A, Cavagna R, Principi N. Hercules Project Collaborative Group. Nasopharyngeal carriage of Streptococcus pneumoniae in healthy children: implications for the use of heptavalent pneumococcal conjugate vaccine. Emerg Infect Dis. 2002;8:479-84.

9. Schettini F Jr, Miragliotta G, Carucci A, Mosca A, Del Vecchio GC, Laforgia N, et al. Nasopharyngeal colonization of Streptococcus pneumoniae in healthy children: percentage of carries, serotypes distribution and antibiotic resistance. Minerva Pediatr. 2003;55(5):439-45.

10. Grupo Multifuncional de Neumonías. Vigilancia epidemiológica centinela de Haemophilus influenzae y Streptococcus pneumoniae en menores de 5 años en el Perú. Rev Per Med Exp Salud Pública. 2003;20(3):150-5.

11. Guevara JM, Aróstegui R, Agurto W, Sobrevilla J, Valencia E, Silva N. Susceptibilidad a antimicrobianos de patógenos respiratorios en niños provenientes de la comunidad. An Fac Med Lima. 2004;65(1):14-8.

12. Guevara JM, Valencia E. Susceptibilidad in vitro de Streptococcus pneumoniae y Moraxella catarrhalis a 5 antimicrobianos orales. Diagnóstico. 2005;44:27-39.

13. Ochoa T, Egoavil M, Reyes I, Castillo M, Chaparro E, Hernández R y col. Grupo Peruano de Investigación en Neumococo. Resistencia antibiótica en enfermedad neumocócica invasiva en pediatría en Lima, Perú. XII Congreso Latinoamericano de Infectología Pediátrica, SLIPE. Costa Rica, May 8-11, 2007.

14. Sener B, Arikan S, Ergin MA, Gunalp A. Rate of carriage, serotype distribution and penicillin resistance of Streptococcus pneumoniae in healthy children. Zentralblatt fur Bakteriologie. 1998;288(3):421-8.

Manuscrito recibido el 30 de enero de 2008 y aceptado para publicación el 15 de febrero de 2008.

Correspondencia:

Dr. José María Guevara Duncan

Instituto de Medicina Tropical Daniel A. Carrión

Jr. José Santos Chocano 199. Bellavista

Callao 2, Perú

Correo-e: jguevarad@unmsm.edu.pe 